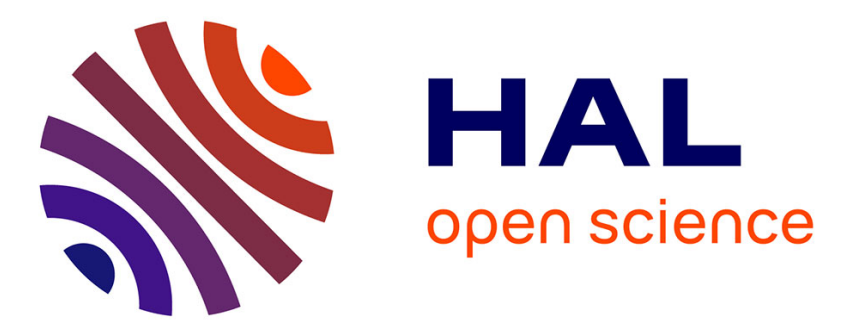

\title{
Influence of the cooperative Jahn-Teller effect on the transport and magnetic properties of $\mathrm{La} 7 / 8 \mathrm{Sr} 1 / 8$ $\mathrm{MnO} 3$ single crystals
}

P. Wagner, I Gordon, S. Mangin, V V Moshchalkov, Y Bruynseraede, L

Pinsard, A. Revcolevschi

\section{To cite this version:}

P. Wagner, I Gordon, S. Mangin, V V Moshchalkov, Y Bruynseraede, et al.. Influence of the cooperative Jahn-Teller effect on the transport and magnetic properties of La $7 / 8$ Sr $1 / 8 \mathrm{MnO} 3$ single crystals. Physical Review B: Condensed Matter and Materials Physics (1998-2015), 2000, 61 (1), 10.1103/PhysRevB.61.529 . hal-02104668

\section{HAL Id: hal-02104668 \\ https://hal.univ-lorraine.fr/hal-02104668}

Submitted on 19 Apr 2019

HAL is a multi-disciplinary open access archive for the deposit and dissemination of scientific research documents, whether they are published or not. The documents may come from teaching and research institutions in France or abroad, or from public or private research centers.
L'archive ouverte pluridisciplinaire HAL, est destinée au dépôt et à la diffusion de documents scientifiques de niveau recherche, publiés ou non, émanant des établissements d'enseignement et de recherche français ou étrangers, des laboratoires publics ou privés. 


\title{
Influence of the cooperative Jahn-Teller effect on the transport and magnetic properties of $\mathrm{La}_{7 / 8} \mathrm{Sr}_{1 / 8} \mathrm{MnO}_{3}$ single crystals
}

\author{
P. Wagner, ${ }^{*}$ I. Gordon, S. Mangin, ${ }^{\dagger}$ V. V. Moshchalkov, and Y. Bruynseraede \\ Laboratorium voor Vaste-Stoffysica en Magnetisme, Katholieke Universiteit Leuven, Celestijnenlaan 200 D, 3001 Leuven, Belgium \\ L. Pinsard and A. Revcolevschi \\ Laboratoire de Chimie des Solides, Université Paris-Sud, 91405 Orsay Cédex, France
}

(Received 12 July 1999)

\begin{abstract}
The low-doped magnetic perovskite $\mathrm{La}_{7 / 8} \mathrm{Sr}_{1 / 8} \mathrm{MnO}_{3}$ undergoes within the paramagnetic-semiconducting phase a first-order structural transition due to antiferrodistorsive ordering of Jahn-Teller deformed $\mathrm{MnO}_{6}$ octahedra. This allows us to study not only the influence of the spin configuration on the magnetotransport properties [colossal magnetoresistance (CMR) effect] but also the role of orbital order and disorder. The orbital ordering transition (at $269 \mathrm{~K}$ in zero magnetic field) causes a doubling of the resistivity (regardless of the CMR effect in applied magnetic fields) and a drop of the paramagnetic susceptibility. The latter might be interpreted in terms of a shrinking of spin polarons. External magnetic fields shift the ordering transition to lower temperatures according to the field-induced decrease of the carrier localization. The magnetic-field-temperature phase boundary line was investigated by means of magnetoresistance (up to $12 \mathrm{~T}$ ) and pulsed-fields magnetization measurements up to $50 \mathrm{~T}$. The pronounced magnetization anomalies, associated with the phase transition, vanish for fields exceeding $20 \mathrm{~T}$. This behavior has been attributed to a field-induced crossover from antiferrodistorsive order to a nondistorsive/ferromagnetic orbital configuration.
\end{abstract}

\section{INTRODUCTION}

Rare-earth $(R)$ manganites with partial divalent $(D)$ substitution on the rare-earth site, i.e., $R_{1-x} D_{x} \mathrm{MnO}_{3}$, show a transition from a paramagnetic-semiconducting phase to a ferromagnetic-quasimetallic state. This insulator-metal transition can be tuned by an external magnetic field, resulting in a negative colossal magnetoresistance (CMR) effect. ${ }^{1}$ An overview on the physics of manganite materials in general and the relevant electronic transitions can be found in the review articles by Ramirez ${ }^{2}$ and by Imada et $a .^{3}$ The relationship between ferromagnetic spin alignment and enhanced charge-carrier mobility between neighboring $\mathrm{Mn}^{3+}$ and $\mathrm{Mn}^{4+}$ ions has been described in terms of the doubleexchange model ${ }^{4}$ and its recent extensions, taking into account the role of electron-phonon coupling and the existence of a Berry phase., ${ }^{5,6}$ Also a Mott-type hopping model, in which the effective barrier depends on the mutual spin orientation at the hopping sites, gives a correct description of the CMR effect in the paramagnetic and in the ferromagnetic state. ${ }^{7}$ The charge carriers are quite close to the localized state and the shielded Coulomb repulsion between them results, for special commensurate substitution ratios, e.g., $x$ $=1 / 8,1 / 4,1 / 2$, in an additional phase transition to a chargeordered, poorly conducting state, being commonly described as "Wigner-" or "'charge-crystal.", $8-10$ Prototypes of charge-ordering compounds are single crystals of $\mathrm{Nd}_{0.5}$ $\left(\mathrm{Pr}_{0.5}\right) \mathrm{Sr}_{0.5} \mathrm{MnO}_{3}$ (Refs. 11 and 12) and $\mathrm{La}_{7 / 8} \mathrm{Sr}_{1 / 8} \mathrm{MnO}_{3}$ (Refs. 13-15). The superstructure of ordered charges (Mn ions of different valency) was in both cases confirmed by neutron- and hard-x-ray diffraction. Fundamentally different, however, is the behavior of these Wigner phases under the influence of external magnetic fields: the 50\%-doped com- pounds are antiferromagnetic insulators at low temperature, and applying magnetic fields destroys the antiferromagnetic alignment as well as delocalizes the charge carriers, thus resulting in a negative magnetoresistance of several orders of magnitude. ${ }^{11}$ At low temperature the $\mathrm{La}_{7 / 8}$ crystals are ferromagnetic insulators, and the transition to the ordered state shifts to higher temperatures under external fields, giving rise to a slightly positive magnetoresistance. ${ }^{14}$

Another important feature of manganites, besides magnetism and charge ordering, is the Jahn-Teller (JT) distortion of the $\mathrm{Mn}^{3+} \mathrm{O}_{6}{ }^{2-}$ octahedra. ${ }^{5}$ A review of the Jahn-Teller effect in general can be found in Ref. 16. Its most common appearance is a volume-conserving elongation of the octahedra along one axis and a compression along the other two axes. This lifts the energetic degeneracy of the $3 d-e_{g}$ level and stabilizes the $3 d_{3 z^{2}-r^{2}}$ orbital with respect to the $3 d_{x^{2}-y^{2}}$ state. In the following we will refer to these orbitals simply as " $3 z^{2}-r^{2}$, and " $x^{2}-y^{2}$." At high temperatures the distortion is not reflected in the variation of the lattice constants due to a mixed occupation of these orbitals, which can moreover be randomly oriented along the main crystalline axes. There is also a breathing-type oscillation between the elongated type and a compressed version of the JT distortion, which favors the $x^{2}-y^{2}$ orbital rather than $3 z^{2}$ $-r^{2}$. This disordered and fluctuating state can be described as a "dynamic JT state,"' abbreviated as DJT. The JTordering temperature $T_{J T}$ is characterized by a preferential occupation of $3 z^{2}-r^{2}$ orbitals and the distortion becomes static with a coherent orientation of the elongated axes throughout the sample. This state is described in the following as "cooperative JT effect" and abbreviated as CJT. Pure $\mathrm{LaMnO}_{3}$ shows the so-called antiferrodistorsive orbital order, ${ }^{17,18}$ which minimizes the increase of elastic energy on 
a macroscopic length scale. This peculiar type of order is preserved in doped manganites with a maximum $\mathrm{Mn}^{4+}$ content of $x=0.15,{ }^{15}$ at the expense of a lowering of $T_{J T}$, e.g., from $790 \mathrm{~K}$ for the undoped system to $269 \mathrm{~K}$ for $x=1 / 8 .^{13,19}$

The main objective of this article is to investigate the relationship between the resistive/magnetic properties of $\mathrm{La}_{7 / 8} \mathrm{Sr}_{1 / 8} \mathrm{MnO}_{3}$ single crystals and the orbital configuration of the $e_{g}$ electrons. These electrons mediate the transport-as well as the magnetic properties - and the difference between a random configuration and a static arrangement of electron orbitals should have a significant impact on charge transfer and magnetic interactions on a macroscopic scale. More specifically, we found that freezing the orbital configuration into a regular pattern enhances the resistivity by roughly a factor of 2 , accompanied by a simultaneous drop of the paramagnetic susceptibility. Both observations will be analyzed and explained on grounds of an orbitalordering model, which is based on the magnetic- and chargetransfer interactions between the diluted $\mathrm{Mn}^{4+}$ ions and the surrounding nearest neighbors of $\mathrm{Mn}^{3+}$.

\section{EXPERIMENT}

Single crystals of $\mathrm{La}_{7 / 8} \mathrm{Sr}_{1 / 8} \mathrm{MnO}_{3}$ were prepared from sintered polycrystalline rods by the floating-zone method, with the growth direction approximately along the $b$ axis. ${ }^{20}$ Due to the relative smallness of the orthorhombic deviation from a perfectly cubic structure we cannot, however, exclude the possibility of some microtwinning in the crystals. The sample used for our measurements was cut to the dimensions $1 \times 2 \times 3 \mathrm{~mm}^{3}$. Four gold contacts were evaporated onto the $2 \times 3 \mathrm{~mm}^{2}$ top side and annealed in the air for $60 \mathrm{~min}$ at $600^{\circ} \mathrm{C}$. The measuring current was flowing in the Lorentzforce free configuration parallel to the applied magnetic field. The magnetoresistivity measurements were performed in a temperature range between $1.5 \mathrm{~K}$ and $300 \mathrm{~K}$ in a cryostat equipped with a superconducting magnet coil generating fields up to $12 \mathrm{~T}$ and magnetization was measured in a superconducting quantum interference device (SQUID) magnetometer in fields up to $5 \mathrm{~T}$. For magnetization studies at higher magnetic fields we employed the pulsed fields setup described in Ref. 21, allowing us to measure magnetization induced by field pulses up to $50 \mathrm{~T}$ on a time scale of $10-20$ $\mathrm{ms}$. The detected signal is hereby the voltage induced in highly sensitive pick-up coils, being proportional to the time derivative of the magnetization $\partial M / \partial t$, which is electronically integrated to $M(B)$. The filling factor and temperaturedependent sensitivity were calibrated according to the absolute magnetization values obtained by the SQUID. The nominal temperatures of these pulsed-field measurements in the considered temperature range (between $200 \mathrm{~K}$ and 300 $\mathrm{K})$ are accurate within $\pm 4 \mathrm{~K}$.

\section{RESULTS AND DISCUSSION}

\section{A. Correlations between the structure and resistive/magnetic properties}

The temperature dependence of the lattice constants in zero external field is given in Fig. 1(a), together with the temperature-dependent resistivity [Fig. 1(b)] and the sample magnetization in Fig. 1(c). The structural data are adopted

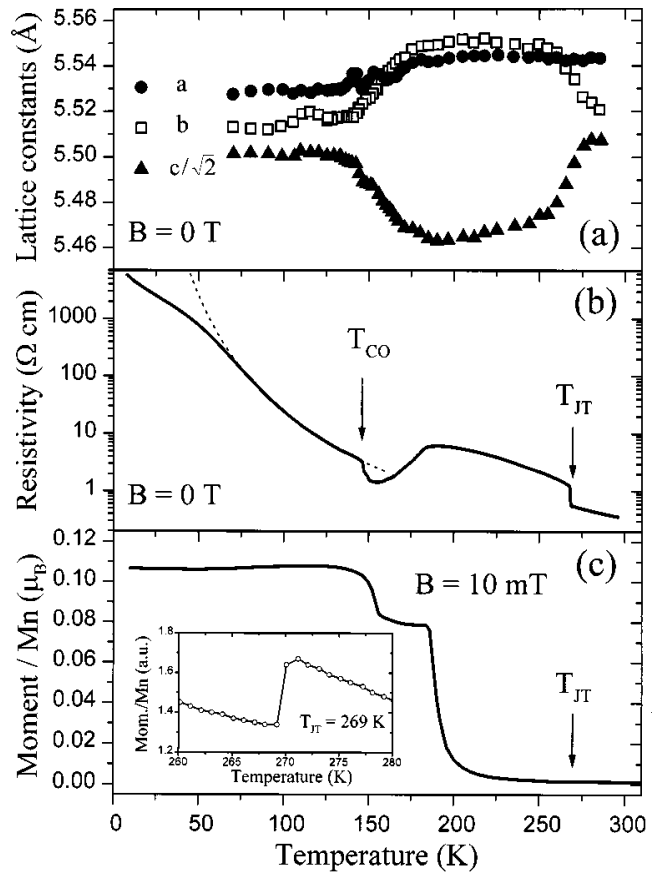

FIG. 1. Temperature dependence of (a) the lattice constants of a $\mathrm{La}_{7 / 8} \mathrm{Sr}_{1 / 8} \mathrm{MnO}_{3}$ single crystal (according to Ref. 15), (b) the resistivity, and (c) the low-field magnetization. The cooperative JT transition at $T_{J T}=269 \mathrm{~K}$ results in a doubling of the resistivity and a drop of the paramagnetic susceptibility [see inset in (c)]. The gradual lifting of the cooperative JT distortion below the Curie temperature $(188 \mathrm{~K})$ is associated with the steplike development of a ferromagnetic/quasimetallic, and finally ferromagnetic/chargeordered state below $T_{C O}=147 \mathrm{~K}$. The dotted line in (b) is a fit to the resistivity increase in the charge-ordered state by ShlovskiiEfros hopping.

from the x-ray and synchrotron radiation studies by Niemöller et al. ${ }^{15}$ and agree with neutron-diffraction results obtained on the same compound by Pinsard et al. ${ }^{13}$ and by Kawano et $a .^{22}$. The structure at room-temperature is pseudocubic with a slight orthorhombic distortion, which becomes notably stronger in the antiferrodistorsive state. The $a$ and $b$ axes are hereby expanded and the $c$ axis becomes compressed. The expansion of $a$ is much less pronounced than the $b$ expansion, which is somewhat uncommon. This might be related to an interplay of the JT distortion with the rotation and tilt of $\mathrm{MnO}_{6}$ octahedra with respect to the $\mathrm{La}_{7 / 8} \mathrm{Sr}_{1 / 8}$ lattice, or to additional lattice distortions induced by the relatively small $\mathrm{Mn}^{4+}$ ions. At low temperatures there is a reentrant structural transition to the very same lattice parameters found at room temperature, and a possible orbital configuration, consistent with these structural data, will be suggested below. The two structural transitions, together with the magnetic transition, allow us to distinguish four different temperature regimes (see Fig. 1).

(i) At room temperature the system is paramagnetic/ semiconducting, and the conductivity is usually ascribed to thermally activated polaron hopping. The JT distortion is not seen in the "macroscopic" structural data, and is therefore of the "disordered" or "dynamic" type. This is schematically shown in Fig. 2, where the $3 d-e_{g}$ electrons of $\mathrm{Mn}^{3+}$ occupy $x^{2}-y^{2}$ as well as $3 z^{2}-r^{2}$ orbitals, oriented along random axes. Moreover, there is a breathing-type phonon 


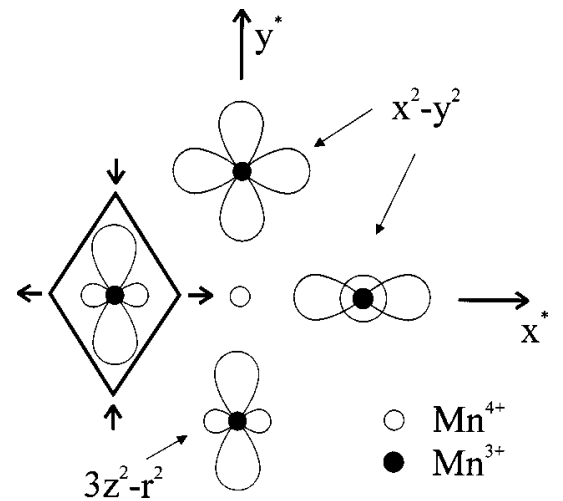

FIG. 2. The dynamic JT state is characterized by a random occupation of $x^{2}-y^{2}$ and $3 z^{2}-r^{2}$ orbitals and a vibronic mode (indicated by arrows at the left $3 z^{2}-r^{2}$ orbital), which favors an oscillation between the two orbital types. The hole-type charge carrier associated with a $\mathrm{Mn}^{4+}$ ion is mobile in three dimensions. The orbitals in this and in the subsequent Figs. 3 and 4 are not drawn on scale with respect to the lattice constant.

mode, which allows for an oscillation between these two orbital types for a given $\mathrm{Mn}^{3+}$ site. The axes in Fig. 2 (and in Figs. 3 and 4) are denoted as $x^{*}, y^{*}, z^{*}$, to discriminate them from those of the pseudocubic unit cell $a, b, c$, and from the $x, y, z$ coordinates, employed for the description of the shape of individual orbitals.

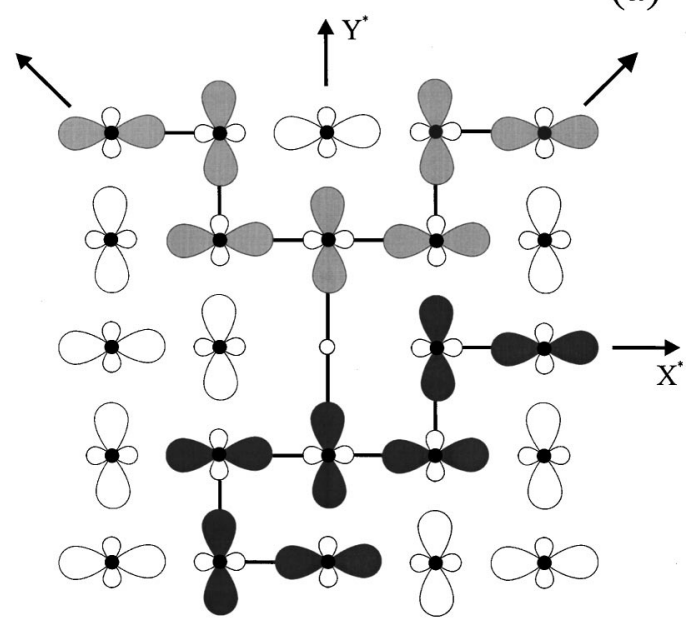

(b)

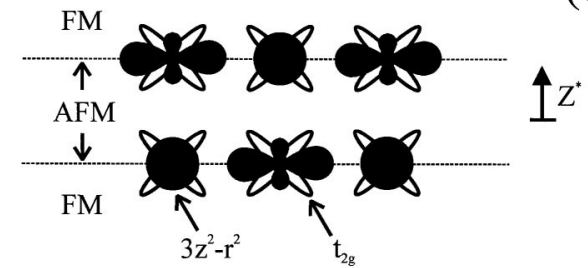

FIG. 3. The antiferrodistorsive order lowers the conductivity along the $x^{*}$ and $y^{*}$ axes by a factor of 2 (possible paths are indicated by dark-shaded orbitals in (a), the conductivity along the diagonal axes remains unaffected (gray-shaded orbitals). The twodimensional character prohibits charge transport along the $z^{*}$ axis (b) and the compression of the crystalline structure along this axis causes $A$-type antiferromagnetism for undoped $\mathrm{LaMnO}_{3}$ via superexchange between the $t_{2 g}$ orbitals.

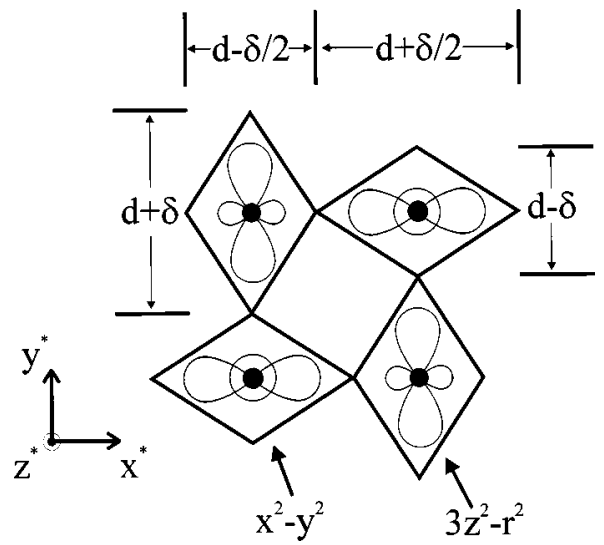

FIG. 4. Tentative orbital structure of slightly doped $\mathrm{LaMnO}_{3}$ at low temperatures. The $x^{2}-y^{2}$ orbitals (50\% occupation) are oriented within the $x^{*} z^{*}$ plane, the $3 z^{2}-r^{2}$ orbitals along the $y^{*}$ axis. The occupation of orbitals in subsequent layers along $z^{*}$ is reversed. The length $d$ corresponds to the mean diameter of $\mathrm{Mn}^{3+} \mathrm{O}_{6}{ }^{2-}$ octahedra without JT distortion. This nondistorsive pattern is able to account for the reentrant structural properties together with ferromagnetic spin alignment, in agreement with the nearestneighbor-coupling rules (Ref. 17).

(ii) At $T=269 \mathrm{~K}$, in the following denoted as Jahn-Teller temperature $T_{J T}$, a structural transition arises, which is attributed to an antiferrodistorsive ordering of JT-elongated $\mathrm{MnO}_{6}$ octahedra, ${ }^{13}$ i.e., the $e_{g}$ electrons occupy predominantly $3 z^{2}-r^{2}$ orbitals. A graphic representation of this peculiar type of orbital order is given in Fig. 3. The expanded pseudocubic axes $a$ and $b$ correspond to the diagonals between $x^{*}$ and $y^{*}$, while the compressed $c$ axis is oriented along the $z^{*}$ direction. This structure of orbitals is equivalent to the "resonant x-ray scattering" results by Murakami et al. on undoped $\mathrm{LaMnO}_{3} .{ }^{18}$ It is noteworthy that the mutual orbital orientation at neighboring $\mathrm{Mn}$ sites causes ferromagnetic correlations within the $x^{*} y^{*}$ plane, and antiferromagnetic superexchange in the perpendicular direction. ${ }^{17}$ In $\mathrm{LaMnO}_{3}$ these interactions are weak and lead to $A$-type antiferromagnetism below $T_{N}=140 \mathrm{~K}$, indicating already that the low-temperature orbital structure of $\mathrm{La}_{7 / 8} \mathrm{Sr}_{1 / 8} \mathrm{MnO}_{3}$ deviates from the antiferrodistorsive type. The orbital ordering at $269 \mathrm{~K}$ is a first-order phase transition (compare the specific-heat results ${ }^{14}$ ) and accompanied by a sudden increase of resistivity (roughly by a factor of 2) and an instantaneous drop of the paramagnetic susceptibility by $20 \%$. These two interrelated effects will be discussed on grounds of the orbital structure in Secs. III C and III D. Effects with similar appearance (enhancement of resistivity and drop of magnetization due to a structural transition) were also observed within the paramagnetic phase of $\mathrm{La}_{0.83} \mathrm{Sr}_{0.17} \mathrm{MnO}_{3}$ (Ref. 23) and in the ferromagnetic phase of $\mathrm{La}_{0.825} \mathrm{Sr}_{0.175} \mathrm{Mn}_{0.94} \mathrm{Mg}_{0.06} \mathrm{O}_{3} \cdot{ }^{24}$ The origin of the structural transition might be different from antiferrodistorsive order due to the relatively high $\mathrm{Mn}^{4+}$ content, exceeding the threshhold value of $15 \% .15$

(iii) From the extrapolation of the inverse paramagnetic susceptibility to zero one finds a ferromagnetic Curie temperature $T_{C}=188 \mathrm{~K}$. At $T_{C}$ the colossal negative magnetoresistance effect is maximum and the resistivity decreases below this temperature in a quasimetallic manner, meaning that 
$d \rho / d T>0$ while the absolute $\rho$ values are unusually high compared to common metals. The antiferrodistorsive orthorhombicity starts to decrease gradually at $T_{C}$ and disappears around $150 \mathrm{~K}$. This indicates that there is a competition between ferromagnetism (induced by the little $\mathrm{Mn}^{4+}$ content) and the antiferrodistorsive structure, favoring an A-type antiferromagnetic (AFM). We note that the magnetization deviates from that of an ideal ferromagnet because the absolute value stabilizes at $3 / 4$ of the low-temperature value. This points to a possible phase separation into ferromagnetic and paramagnetic or antiferromagnetic areas-a phenomenon that is observed in a wide variety of manganite compounds. ${ }^{25}$

(iv) At $147 \mathrm{~K}$ the resistivity increases spontaneously by roughly a factor of 2 , which is associated with a chargeordering transition to a Wigner-crystal state. The charge order was demonstrated by the observation of superlattice reflections in $\mathrm{x}$-ray diffraction. ${ }^{15}$ The charge-ordering transition is also accompanied by a sudden jump in magnetization to the actual low-temperature value and by the vanishing of the macroscopically observed JT distortion. The Wigner state is not perfectly insulating and the resistivity increase between $146 \mathrm{~K}$ (right below the Wigner transition) and $75 \mathrm{~K}$ can best be described by Shklovskii-Efros (SE) hopping with $\rho(T) \propto \exp \left\{\left(T_{0} / T\right)^{1 / 2}\right\} .{ }^{26}$ This hopping mechanism corresponds to variable range hopping with a soft Coulomb gap in the density of states. Other hopping processes, like thermally activated (polaron) hopping, Mott's variable range hopping, or cascade hopping, do not apply. From the SE description of the resistivity in the temperature regime between $75 \mathrm{~K}$ and $146 \mathrm{~K}$ [see fit function in Fig. 1(b)] we could extract $T_{0}=1.26 \times 10^{4} \mathrm{~K}$, where $T_{0}$ is given by $k_{B} T_{0}$ $=2.8 e^{2} /\left(4 \pi \epsilon_{0} \epsilon_{L} L\right)$, with $\epsilon_{L}$ being the dielectric constant of the lattice and $L$ the carrier localization length. ${ }^{26}$ The resulting $L=470 \AA / \epsilon_{L}$ seems of the correct magnitude, since the dielectric constant of the ionic perovskites easily achieves values in the range of $10^{2}$, e.g., up to 300 for the isostructural $\mathrm{SrTiO}_{3}$. More precise data on $\epsilon_{L}$ of manganites are to our knowledge not yet available. The resistivity increase below $75 \mathrm{~K}$ is weaker than described by any of the aforementioned hopping processes, including SE hopping. The finite conductivity for $T \rightarrow 0$ means that the Wigner crystal exhibits imperfections in form of nonlocalized charge carriers, which might result from slight deviations from the exact 1/8 doping ratio. Interestingly enough, this remanent conductivity is also found in the charge-ordered Pr- and Nd-manganites with $50 \%$ strontium doping. ${ }^{11,12}$ The resistivity increase below 40 $\mathrm{K}$ [Fig. 1(b)] scales with $-\ln T$, pointing either to a Kondotype problem or to a resistivity contribution caused by electron-electron interactions. ${ }^{27}$

In the low-temperature limit we found a magnetic moment per Mn ion of $3.75 \mu_{B}$ (at $10 \mathrm{~K}, 5 \mathrm{~T}$ ). This is very close to the spin-only value for a mixture of $\mathrm{Mn}_{7 / 8}{ }^{3+}(J=s=2)$ and $\mathrm{Mn}_{1 / 8}{ }^{4+}(J=s=3 / 2)$ and a gyromagnetic ratio of $g$ $=2$, resulting in $3.88 \mu_{B}$. This is, within the precision of the measurement, compatible with ferromagnetic spin alignment, although neutron diffraction gave possible evidence for a small spin canting. ${ }^{13}$

Both observations-ferromagnetism and recovery of the DJT-lattice parameters-suggest that the orbital structure at low temperatures is different from the antiferrodistorsive configuration. The possible guess that lowering temperature transfers the static order back to a dynamic/disordered JT state, as at high temperatures, is clearly contraintuitive. Furthermore, XAFS studies on $\mathrm{La}_{2 / 3} \mathrm{Ca}_{1 / 3} \mathrm{MnO}_{3}$ have shown that there are two peaks in the distribution function of $\mathrm{Mn}-\mathrm{O}$ bond lengths for the paramagnetic state (attributed to the JT distortion), which merge to a single peak for the ferromagnetic quasimetal. ${ }^{28}$ This is interpreted in the sense that the $e_{g}$ electrons become delocalized and need therefore not to profit from the formation of JT distortions on the scale of individual unit cells. In the case of $\mathrm{La}_{7 / 8} \mathrm{Sr}_{1 / 8} \mathrm{MnO}_{3}$ at low temperatures, the electrons are, despite ferromagnetism, well localized and the JT-distortion is nevertheless not seen in the x-ray-diffraction (XRD) data of Fig. 1(a). This may be interpreted in terms of the orbital structure sketched in Fig. 4, which agrees with a " $G$-type orbital order," in the scheme of Maezono et al. ${ }^{29}$ and Endoh et al. ${ }^{30}$ First, this orbital configuration results in a ferromagnetic nearest-neighbor coupling between $\mathrm{Mn}^{3+}$ ions for all directions, ${ }^{17}$ while the coupling between $\mathrm{Mn}^{3+}$ and the low-concentrated $\mathrm{Mn}^{4+}$ is per se mainly ferromagnetic. Second, this structure provides an exact compensation of the elongated and compressed axes of the JT-distorted octahedra on a macroscopic scale: the $3 z^{2}-r^{2}$ orbitals (50\% occupation) are surrounded by octahedra, which are elongated by a value $+\delta$ along the orbital's axis. Since the JT effect is in first approximation volumeconserving, the contraction along the two perpendicular axes has to be $\approx-\delta / 2$. The corresponding figures for the $x^{2}-y^{2}$ orbitals ( $50 \%$ occupation) are an expansion by $+\delta / 2$ within the plane of these orbitals, and a compression by $-\delta$ in the perpendicular direction. These distortions compensate precisely, in the structure of Fig. 4, along the three spatial directions, provided that we can ignore local distortions caused by the low concentrated $\mathrm{Mn}^{4+}$.

\section{B. Influence of the CJT transition on the resistivity}

We performed $\rho(B)$ measurements at constant temperatures around $T_{J T}$, see Fig. 5. Besides the usual CMR behavior, there is a sharp negative magnetoresistance effect upon crossing the boundary of the antiferrodistorsive phase, which is stable in low fields, and entering the disordered DJT phase in higher magnetic fields. The strong hysteresis effect (being especially pronounced at $268 \mathrm{~K}$ ) gives further confirmation of the first-order nature of the CJT transition. Upon lowering the temperature the transition shifts to higher fields with a shrinking of the hysteresis width. The shape of the phaseboundary line will be discussed in Sec. III D. Almost independent of the temperature is, however, the relative height of the resistive jump with $2.05 \pm 0.15$, shown as solid dots in Fig. 5. This result was corroborated by $\rho(T)$ measurements at constant fields, and the resulting jump heights are given in Fig. 5 by open circles. At present it seems difficult to give an unambiguous explanation for the doubling of $\rho$ upon the CJT transition since several possibilities seem to apply equally well.

\section{Change of the double-exchange overlap integral}

It was argued ${ }^{24}$ that the JT elongation of $\mathrm{MnO}_{6}$ octahedra and the exclusive occupation of the $3 z^{2}-r^{2}$ orbitals might lower the double-exchange overlap integral along the elongated axis, while the overlap integrals with neighbors along 


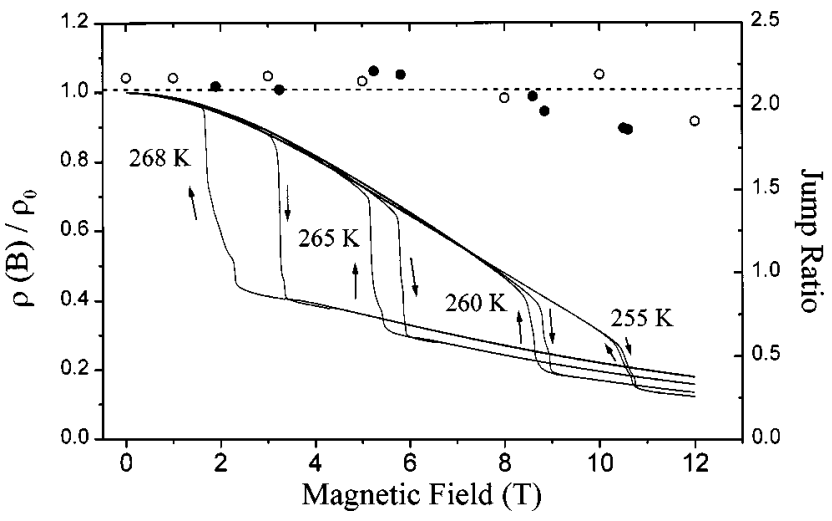

FIG. 5. Normalized magnetoresistance (left axis) at the transition from the cooperative JT state in low fields to the dynamic JT state in higher fields. Upon lowering the temperature the width of the hysteresis loops decreases rapidly, indicating a weakening of the first-order character of the CJT transition under the presence of external magnetic fields. The relative height of the resistive jump from the ordered to the disordered state (right axis) is almost field and temperature independent with an absolute value around 2.1. Solid dots refer to the magnetoresistance measurements at constant temperature, open dots refer to temperature sweeps at fixed magnetic fields. The dotted line is a guide to the eye.

the perpendicular axes should become negligible. This is somewhat doubtful, because JT distortions, albeit fluctuating and in random directions, are present already above $T_{J T}$. Furthermore, the change in orthorhombicity at $T_{J T}$ might bring about a spontaneous modification of the bond angle between $\mathrm{Mn}^{3+}, \mathrm{O}^{2-}$, and $\mathrm{Mn}^{4+}$. The importance of this angle for the absolute resistivity values (again via the overlap integral) was pointed out in Ref. 31. Neutron diffraction on undoped $\mathrm{LaMnO}_{3}$, however, has proven that the tilt angle of $\mathrm{MnO}_{6}$ octahedra (responsible for the buckling of the MnO-Mn bonds) indeed increases with decreasing temperature-but there is no discontinuous change around $T_{J T}{ }^{19}$

\section{Decrease of the carrier localization volume}

Alternatively, we can consider a transport mechanism on the basis of Mott's hopping concept, which is suggested by the low carrier mobility resulting from a strong carrier localization. ${ }^{32,7}$ The resistivity in this model (and related hopping concepts) depends exponentially on the ratio between the average hopping distance $R$ (at high temperatures corresponding to the nearest-neighbor distance) and the half of the carrier localization length $L$. According to the orbital structure sketched in Fig. 2, we might assume a localization of the hole-type carrier within a volume $V_{L}$ extending from the $\mathrm{Mn}^{4+}$ ion to the six nearest neighbors (via hybridization of orbitals) for the DJT state. The CJT ordering (Fig. 3) will restrict $V_{L}$ to the $\mathrm{Mn}^{4+}$ site and only two out of the six nearest neighbors. From this we can calculate for both phases a localization length averaged over the three main crystallographic directions, resulting in a resistive jump ratio of $\approx 1.70$. We point out that this approach has to average over actually anisotropic localization lengths, a situation for which the Mott-hopping mechanism is conceptually not yet worked out.

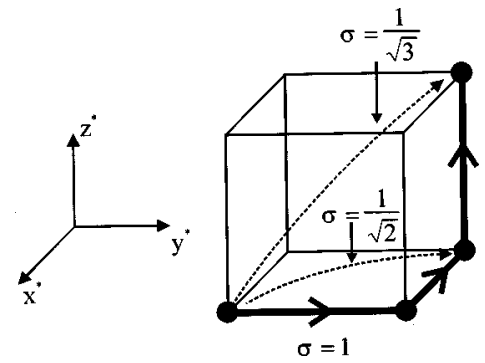

FIG. 6. Illustration of nearest-neighbor hopping on a cubic network with different conductivities for principal axes, square diagonals, and cube diagonals. The elemental conductivities $(\sigma$ $=1,1 / \sqrt{2}, 1 / \sqrt{3}$ ) have to be weighted by the number of possible paths, and by the probability for a suitable orbital-overlap configuration.

\section{Frustration of charge transport by orbital ordering}

The doubling of the resistivity may be understood by considering the change in the allowed hopping paths in Figs. 2 and 3. We postulate that charge transport between $\mathrm{Mn}^{3+}$ and $\mathrm{Mn}^{4+}$ can only occur in configurations with orbital overlap. This means that an $e_{g}$ electron in a $x^{2}-y^{2}$ orbital can only be transferred to nearest-neighbor $\mathrm{Mn}^{4+}$ ions, which are located in the plane of this orbital, but not in the perpendicular direction. An $e_{g}$ electron in a $3 z^{2}-r^{2}$ orbital can be transferred to nearest-neighbor $\mathrm{Mn}^{4+}$ ions located on the axis of this orbital, but not in the equatorial directions. We attribute to both processes an equal, dimensionless conductivity $\sigma$ $=1$, and a possible magnetic impedence of the charge transfer by spin misalignment will preliminarily be ignored.

In the disordered state (Fig. 2) the hole associated with $\mathrm{Mn}^{4+}$ can move to all nearest $\mathrm{Mn}^{3+}$ neighbors (due to low doping we consider only $\mathrm{Mn}^{3+}$ ), which provide orbital overlap, and is in principal mobile in three dimensions. The probability for orbital overlap is, regardless of the breathing mode and depending only on the disordered nature of the orbital arrangement, given by the factor 1/2: $50 \%$ of the $e_{g}$ electrons occupy $3 z^{2}-r^{2}$ orbitals, which point with a $1 / 3$ probability into the direction of a given nearest-neighbor site. The other $50 \%$ of $e_{g}$ electrons occupy $x^{2}-y^{2}$ orbitals with a respective statistical weight of $2 / 3$. The antiferrodistorsive order (Fig. 3) confines the charge carrier within the $x^{*} y^{*}$ plane (movement perpendicular to this plane is forbidden by the lack of suitable orbitals), and direct charge transfer within this plane can occur only to two out of the four nearest neighbors.

Furthermore, we need to take into account that the sample exhibits microtwinning in the sense that the current path probes equal portions of differently oriented domains. Therefore we calculate the relative conductivity averaged along arbitrary directions of a three-dimensional cubic network, assuming that domains with various orientations contribute to the total conductivity like resistors connected in parallel. The average of conductivities can be approximated by the average of the three types of directions shown in Fig. 6: (i) the principal axes of the crystal $\left(x^{*}, y^{*}\right.$, and $z^{*}$ are used in the sense of Fig. 2); (ii) the square diagonals $\left(x^{*} y^{*}, x^{*} z^{*}\right.$, and $\left.y^{*} z^{*}\right)$; (iii) the cube diagonals.

In the three-dimensional DJT state the conductivity of a $\mathrm{Mn}^{4+}$ hole along the principal axes is $\sigma_{p}^{3 d}=1 / 2$, i.e., the 
elemental conductivity $\sigma=1$ ( 1 unit-cell distance is spanned by 1 hopping event) is weighted with the probability for a suitable orbital geometry.

The elemental conductivity along square diagonals is $1 / \sqrt{2}$ ( 2 hops are required for a distance of $\sqrt{2}$ unit cells), and we take into account that there are two possible hopping paths with a respective probability for orbital overlap of $(1 / 2)^{2}$. The square-diagonal conductivity is therefore $\sigma_{s d}^{3 d}$ $=1 / \sqrt{2} \times 2 \times(1 / 2)^{2} \approx 0.354$.

Correspondingly, we obtain for cube diagonals $\sigma=1 / \sqrt{3}$ ( 3 hops for a distance of $\sqrt{3}$ unit cells), and there are now six possible paths with a probability factor of $(1 / 2)^{3}$. The cubediagonal conductivity is then $\sigma_{c d}^{3 d}=1 / \sqrt{3} \times 6 \times(1 / 2)^{3}$ $\approx 0.433$.

The arithmetic average of $\sigma_{p}^{3 d}, \sigma_{s d}^{3 d}$, and $\sigma_{c d}^{3 d}$ approximates the averaged conductivity along all possible directions and has a numerical value of $\overline{\sigma^{3 d}} \approx 0.429$.

The major difference in the more two-dimensional situation with antiferrodistorsive order is the suppression of conductivity along the $z^{*}$ direction. Considering the conductivity along the two remaining principal axes we note that, in the average, two steps are required to span a distance of one unit cell in the desired direction, i.e., the conductivity is reduced by a factor of 2 . This is indicated by thin solid lines along the black-shaded orbitals in Fig. 3. The corresponding average conductivity is $\sigma_{p}^{2 d}=(1 / 3) \times(0+1 / 2+1 / 2)=1 / 3$. Due to the static orbital arrangement we do not need to consider the probability factors for overlap geometries. The number of steps for charge transport along the diagonal direction $x^{*} y^{*}$ is not altered by the orbital ordering (see diagonal lines along the gray-shaded orbitals in Fig. 3), however the conductivity along the other two square-diagonal directions becomes zero. The average square-diagonal conductivity is therefore $\sigma_{s d}^{2 d}=1 / 3 \times(\sqrt{2} / 2+0+0) \approx 0.236$. It is evident that the cube-diagonal conductivity $\sigma_{c d}^{2 d}$ is 0 and the total average of all directions results in $\overline{\sigma^{2 d}} \approx 0.190$.

The relative resistive jump height at the CJT transition is then given by the ratio $\overline{\sigma^{3 d}} / \overline{\sigma^{2 d}} \approx 2.26$, in very close agreement with the experimentally found jump ratios between 1.9 and 2.2 (slightly dependent on the applied magnetic field). We note that magnetoresistive measurements in untwinned single crystals, with the current path along well-defined crystallographic axes, should bring about jump ratios different than 2.

\section{Influence of the CJT transition on the paramagnetic susceptibility}

Associated with the doubling of the resistivity, we found also a sudden drop in the paramagnetic magnetization, which is shown in the insert of Fig. 1(c). In field-dependent magnetization measurements at constant temperatures (up to $5 \mathrm{~T}$ with a SQUID magnetometer, and up to $50 \mathrm{~T}$ in pulsed magnetic fields, see Figs. 7 and 8) this corresponds to a sudden, hysteretic upturn of the magnetization upon leaving the CJT state, becoming unstable in sufficiently high fields. For a better comparison, Fig. 7 includes also a magnetization curve at $272 \mathrm{~K}$, i.e., above the JT-ordering temperature, and a magnetization curve at $265 \mathrm{~K}$, i.e., entirely inside the ordered phase. In addition to the presented $M(B)$ data we studied

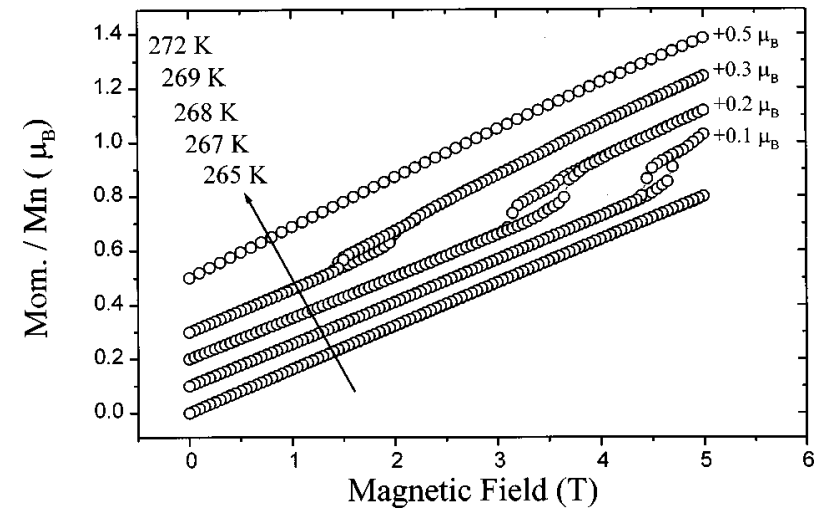

FIG. 7. SQUID magnetization measurements around the CJT transition: the orbitally ordered state becomes unstable at higher fields, resulting in a hysteretic upturn of the paramagnetic magnetization. For clarity, the absolute values of the magnetization curves at different temperatures are shifted by the indicated offset values.

magnetization curves at various temperatures between $220 \mathrm{~K}$ and $290 \mathrm{~K}$. The signature of the CJT transition is especially pronounced for the pulsed-fields magnetization technique (Fig. 8). As a general tendency we can note that at lower temperatures (or respectively: at higher magnetic fields) the hysteresis width shrinks rapidly and the relative upturn of the magnetization decreases. Below $235 \mathrm{~K}$ and a corresponding field of $18 \mathrm{~T}$, we had no indication for a further distinction between a JT-ordered and a disordered state.

To understand the influence of the CJT transition on the magnetic properties, we should analyze the paramagnetic magnetization and susceptibility data not only in the framework of a simple model with independent magnetic ions, but take also into account the existence of spin polarons. The "spin polaron" denotes here an ensemble of neighboring unit cells with parallel (on a local scale ferromagnetic) spin alignment, which leads to a "superparamagnetic behavior." The existence of these clusters in CMR manganites was postulated in Ref. 33, and found an experimental support in Refs. 7, 34, and 35. In compounds with negligible orbital order, i.e., with higher-doping concentration, the charge transport is governed by the relative spin misorientation be-

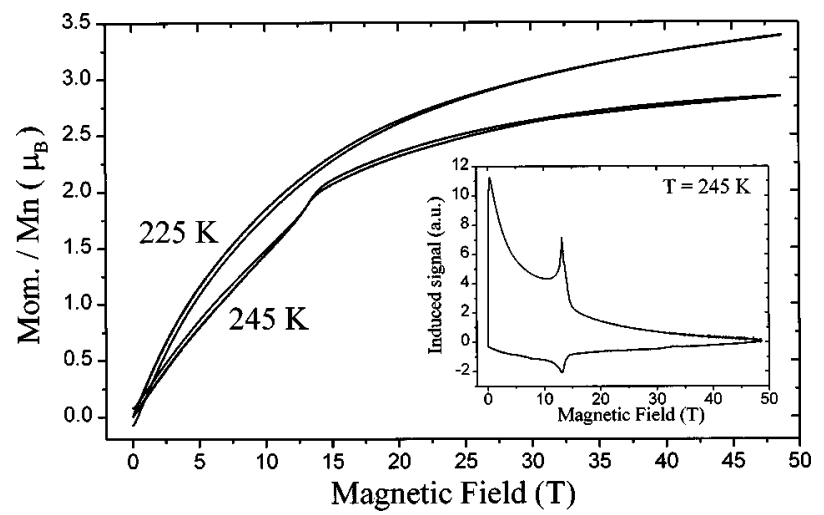

FIG. 8. The pulsed-fields magnetization measurement at $245 \mathrm{~K}$ is qualitatively equivalent to the curves in Fig. 7. The signature of the phase transition vanishes abruptly for temperatures lower than $235 \mathrm{~K}$ (here shown for $225 \mathrm{~K}$ ). The orbital-ordering transition results in pronounced spikes in the nonintegrated $d M / d B$ signal (see inset). 


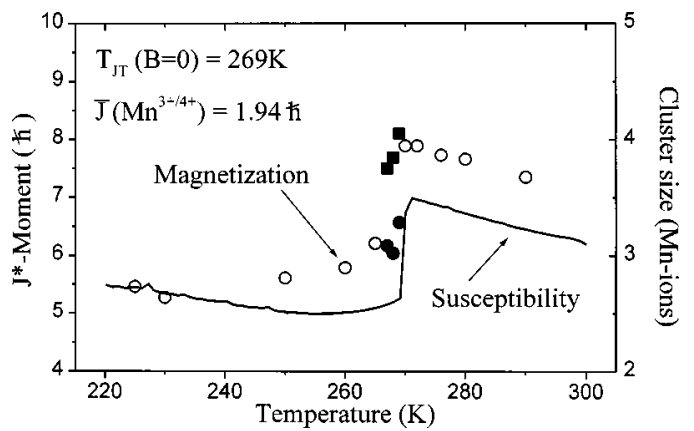

FIG. 9. The temperature-dependent size of preformed ferromagnetic spin clusters in the paramagnetic phase around the CJT transition. The data points were determined by Brillouin fits to the magnetization curves (Fig. 7) and suggest a shrinking from four to three $\mathrm{Mn}$ ions involved in the formation of an individual spin cluster. Measurements crossing the phase-boundary line gave two corresponding data points (solid squares and circles). The solid line was calculated on the basis of the low-field magnetization data from Fig. 1(c).

tween neighboring spin polarons, while the carriers are delocalized within their respective spin cluster. ${ }^{7}$

For the size determination of spin polarons from the fielddependent magnetization data (SQUID results in Fig. 7) we employed first a fitting based on the Brillouin function $\mathcal{B}$, which is justified since the respective temperatures around $T_{J T}(B=0)$ are sufficiently above the ferromagnetic transition. The paramagnetic magnetization along the field axis (per unit-cell volume) is usually given by $M(B, T)$ $=g \mu_{B} J \mathcal{B}\left\{g \mu_{B} J B / k_{B} T\right\}$, with the gyromagnetic ratio $g=2$, $\mu_{B}$ the Bohr magneton, and $J$ the spin moment of the magnetic ions. ${ }^{36}$ For the mixture of $\mathrm{Mn}^{3+}$ and $\mathrm{Mn}^{4+}$, we replace $J$ by the average $\bar{J}=1.94$. In the case of superparamagnetic clusters composed of $n$ ions, the $J$ factor in the argument of the Brillouin function changes to $J^{*}=n \bar{J}$ (causing a modification of the curvature), while the $\bar{J}$ in the prefactor remains unchanged. This is due to the compensation of the $n$-fold increase in magnetic moment per (super) paramagnetic entity by the $1 / n$-fold decrease of their density per unit volume. Finally, we have to replace the temperature $T$ by the effective temperature scale $\left(T-T_{C}\right)^{\gamma}$, with $T_{C}=188 \mathrm{~K}$. Since we are not too close to $T_{C}$ it is reasonable to choose $\gamma=1$, according to the Curie-Weiss law. ${ }^{36}$

The result of the fitting is given in Fig. 9 and suggests a CJT-induced shrinking of superparamagnetic clusters from $J^{*}=8\left(\approx 4 \mathrm{Mn}\right.$ ions envolved) to $J^{*}=6$ (corresponding to roughly $3 \mathrm{Mn}$ spins). The three magnetization curves measured across the CJT phase boundary gave hereby six data points. Besides this shrinking there is a slight tendency towards an increasing cluster size with decreasing temperature. Describing these cluster sizes on the grounds of the orbital structures is difficult, since a random orientation of $e_{g}$ electrons results, in principle, in ferromagnetic nearest-neighbor correlations, while the antiferrodistorsive structure promotes $A$-type antiferromagnetism. ${ }^{17}$ These interactions are, however, weak because already in the case of pure $\mathrm{LaMnO}_{3}$, where CJT order is established around $800 \mathrm{~K}$, the $A$-type AFM spin order is only observed far below room temperature. We will therefore restrict the discussion to the strongest ferromagnetic bonds, i.e., the coupling of the diluted $\mathrm{Mn}^{4+}$

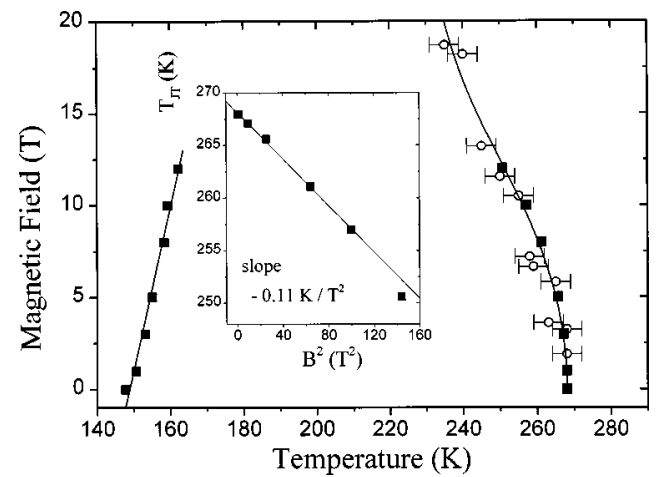

FIG. 10. Increasing magnetic fields shift the CJT transition to lower temperatures (quadratic in low fields, compare inset) and the charge-ordering transition to higher temperatures (the dotted line is a guide to the eye). The orbital-ordering line is fitted on grounds of the field-induced mobility contribution to the free energy of the DJT state. The distinction between the orbitally ordered and disordered state ceases above $20 \mathrm{~T}$. The squares correspond to resistive data from $\rho(T)$ measurements at constant fields, the open circles to pulsed-field magnetization.

ions to the neighboring $\mathrm{Mn}^{3+}$ sites. In the case of the ordered structure (see Fig. 3), the central $\mathrm{Mn}^{4+}(J=3 / 2)$ can undergo spin alignment in the sense of double exchange with 2 out of the $6 \mathrm{Mn}^{3+}$ neighbors $(J=2)$. The total $J^{*}$ moment of this entity corresponds to 5.5. For the DJT state (Fig. 2), 3 out of the $6 \mathrm{Mn}^{3+}$ neighbors are, on the average, in the $x^{2}$ $-y^{2}$ configuration, and the probability that an orbital lobe points towards the central $\mathrm{Mn}^{4+}$ is $2 / 3$. The other 3 orbitals are of the $3 z^{2}-y^{2}$ shape, and the overlap probability is $1 / 3$ (according to the three possible spatial orientations of these orbitals). The average total moment of this entity is therefore 7.5. It might be accidental, but these figures (5.5 and 7.5) agree closely with the experimentally found $J^{*}$ values in the CJT and DJT phases.

As an alternative method for the determination of cluster sizes we analyzed also the low-field magnetization data from Fig. 1(c) by means of the modified (superparamagnetic) susceptibility formula $\chi=\left(g \mu_{B}\right)^{2} \bar{J}\left\{\left(J^{*}+1\right) /\left[3 k_{B}\left(T-T_{C}\right)\right]\right\}{ }^{36}$ The resulting $J^{*}$ values (solid line in Fig. 9) are similar to the aforementioned data, suggesting that the size of these clusters is quite insensitive to the influence of external fields. From the findings of this subsection we conclude that each hole-type charge carrier associated with a $\mathrm{Mn}^{4+}$ ion is embedded into a locally ferromagnetic environment, extending to the nearest-neighbor sites. The charge transfer depends, therefore, on the orbital configuration between $\mathrm{Mn}^{4+}$ and its nearest neighbors, while a possible impedence of the charge movement by magnetic disorder might, in a first approximation, be ignored.

\section{Competition between paramagnetic spin alignment and orbital ordering}

It can be seen from Fig. 10 that increasing magnetic fields shift the cooperative JT transition, identified by the resistive jump and the susceptibility drop, to lower temperatures. This shift is quadratic in moderate fields (see inset of Fig. 10) with a slope of $-0.11 K / T^{2}$, in agreement with the behavior published in Ref. 14. It was argued in an earlier work on double exchange that ferromagnetic spin alignment enhances the mobility of charge carriers and lowers thereby their ki- 
netic energy in the sense of the uncertainty principle. ${ }^{4}$ The possible energy decrease due to itinerant behavior can, certainly in a ferromagnetic/quasimetallic state, exceed the total energy decrease associated with a localized state forming a JT-deformed environment. ${ }^{28}$ Here we are dealing with a similar problem.

In the higher-conducting DJT state the carriers are relatively mobile and charge transfer is impeded by spin disorder. This spin disorder persists in the CJT state, however, the static orbital structure results in an additional carrier localization, as discussed in Sec. III B. The enhanced kinetic energy of the carrier system is hereby overcompensated by the elastic energy of the lattice, which is responsible for the transition to the antiferrodistorsive structure. While external magnetic fields lower the degree of spin-disorder localization in the CJT state and in the DJT state, the gain in free energy is more pronounced in the latter case. The frustration of carrier mobility in the CJT state would even persist in the absence of spin disorder due to the static orbital arrangement. In conclusion, magnetic fields lower the kinetic energy of the carrier system more effectively in the DJT state than in the CJT state, and the DJT state becomes stable in a wider temperature range, meaning that the orbital-ordering transition shifts to lower temperatures.

Possible measures of the field-induced decrease of free energy in the DJT state are the enhancement of carrier mobility, and equivalently, the decrease of resistivity. The CMR effect scales, in the paramagnetic state, with the square of the Brillouin function, depending on the total moment of superparamagnetic entities, ${ }^{7}$ and the field-dependent transition temperature $T_{J T}(B)$ should scale according to the implicit equation:

$$
T_{J T}(B)=T_{J T}(B=0)-\alpha \mathcal{B}^{2}\left(\frac{g \mu_{B} J^{*} B}{k_{B}\left[T_{J T}(B)-T_{C}\right]}\right) .
$$

The $J^{*}$ moment was chosen with eight (experimental value right above the phase transition in Fig. 9), and $\alpha$, the only free parameter, connects the decrease of free energy (via delocalization) to the CMR-induced lowering of the resistivity. The equation was solved numerically for $T_{J T}(B)$ and the best agreement with the data (see the solid line Fig. 10) was achieved for $\alpha=47 \mathrm{~K}$, with an uncertainty of $\pm 2 \mathrm{~K}$. The shape of the phase-boundary line is correctly reproduced and the quadratic low-field behavior emerges directly from the properties of the Brillouin function. There are two alternative mechanisms that can also explain a decrease of $T_{J T}$ with $B^{2}$, but these effects are probably too small to account for the observed shift.

First, the intrinsic antiferromagnetism of the antiferrodistorsive structure is caused by superexchange due to the overlap of $t_{2 g}$ orbitals along the $z^{*}$ axis (Fig. 3). External magnetic fields, or an internal Weiss field in the ferromagnetic state, should therefore result in a decompression of the $z^{*}$ axis in order to minimize the superexchange interaction, which causes, in turn, a destabilization of the antiferrodistorsive order. The correlations from superexchange are, however, weak and only notable below $T_{N} \approx 0.5 \times T_{J T}$.

Second, it is known that magnetic fields cause a shrinking of the exponentially falling tails of electron-wave functions. ${ }^{37}$ This shrinking reduces the overlap between the
$3 z^{2}-r^{2}$ orbital and the two opposite $\mathrm{O}^{2-}$ orbitals and allows therefore for smaller JT distortions, with less-pronounced minima in the free energy. The compression of the Bohr radius $a$ is given by $a \rightarrow a\left[1-a^{4} /\left(24 \lambda^{4}+3 a^{4}\right)\right]$, with $\lambda$ being the "Larmor length" $(\hbar / e B)^{1 / 2}{ }^{37}$ This expression is valid for $a \ll \lambda$ and predicts essentially a shrinking proportional to the square of the local magnetic field. Comparing the Bohr radius of $e_{g}$ electrons $(\approx 2 \AA)$ with the Larmor length at $50 \mathrm{~T}(36 \AA)$ corresponds to a compression effect of the order of $10^{-7}$, which is insufficient to affect the JT distortion itself.

It is noteworthy that the CJT transition vanishes for temperatures below $235 \mathrm{~K}$ (see also Fig. 8), meaning that it might become second order. The charge-ordering transition, characterized by the lifting of the antiferrodistorsive structure and by ferromagnetic spin alignment, shifts in nonzero fields to higher temperatures, because the internal Weiss field is corroborated by the external contribution, see Fig. 10. This means that sufficiently high fields can stabilize the nondistorsive orbital structure (Fig. 4) already at temperatures above $T_{c o}=147 \mathrm{~K}$. We speculate that for temperatures where the CJT transition apparently vanishes, see, e.g., the pulsedfields measurement at $225 \mathrm{~K}$ in Fig. 8, the increasing external field can transform the antiferrodistorsive order gradually to the nondistorsive type. If there is a phase mixture between these two states, the typical signatures of the CJT transition in magnetization and resistivity will be smeared out and finally vanish, because transport and magnetic properties should be widely similar in the nondistorsive state and in the disordered JT state.

\section{CONCLUSIONS AND SUMMARY}

We investigated the resistive and magnetic behavior of a rare-earth manganite in the low-doping regime, $\mathrm{La}_{7 / 8} \mathrm{Sr}_{1 / 8} \mathrm{MnO}_{3}$, where the high- $\mathrm{Mn}^{3+}$ content results in a cooperative Jahn-Teller effect. The phase transition from the orbitally disordered state to a phase with antiferrodistorsive orbital order has a substantial influence on the resistive as well as on the magnetic properties. The orbital ordering impedes charge transfer along certain directions, resulting in a doubling of the resistivity irrespective of external magnetic fields and the corresponding spin-alignment and CMR effects. The magnitude of this resistive jump was calculated and found to be quantitatively correct on the basis of a simple model, and crossing the phase boundary from the orbitally ordered to the disordered state corresponds to a strong, negative magnetoresistance effect, which is independent of the CMR effect as such. The lower resistivity of the disordered state goes along with an increase of the paramagnetic susceptibility, which is especially remarkable, since it can only be explained on grounds of superparamagnetic behavior controlled by ferromagnetic spin clusters or spin polarons. The typical cluster moment was evaluated by two independent fitting procedures and corresponds to the total moments of $\mathrm{Mn}^{4+}$ ions and the surrounding $\mathrm{Mn}^{3+}$ sites, to which the $\mathrm{Mn}^{4+}$ has an orbital overlap. The shift of the orbital-ordering transition to lower temperatures under the influence of magnetic fields was explained by a stabilization of the disordered phase through an enhancement of the carrier mobility. Furthermore, we pointed out that the lowtemperature properties of $\mathrm{La}_{7 / 8} \mathrm{Sr}_{1 / 8} \mathrm{MnO}_{3}$, which are incom- 
patible with antiferrodistorsive order, might be explained on grounds of a static_-but nondistorsive-orbital structure.

\section{ACKNOWLEDGMENTS}

This work was supported by the Flemish Concerted Action (GOA), the Fund for Scientific Research-Flanders
(FWO), and the Belgian Interuniversity Attraction Poles programs (IUAP). The authors thank L. Trappeniers for technical support with the pulsed-fields magnetization measurements and V. Bruyndoncx for help with the numerical solution of the phase-boundary line. Constructive advice by R. Gross, S. Uhlenbruck, and B. Büchner from the University of Cologne is gratefully acknowledged.
*Electronic address: Patrick.Wagner@fys.kuleuven.ac.be

†Present address: Laboratoire de Physique des Matériaux, Université H. Poincaré, Boîte Postale 239, 54506 Vandoeuvre-les-Nancy Cédex, France.

${ }^{1}$ R. M. Kusters, J. Singleton, D. A. Keen, R. McGreevy, and W. Hayes, Physica B 155, 362 (1989); R. von Helmolt, J. Wecker, B. Holtzapfel, L. Schultz, and K. Samwer, Phys. Rev. Lett. 71, 2331 (1993).

${ }^{2}$ A. P. Ramirez, J. Phys.: Condens. Matter 9, 8171 (1997).

${ }^{3}$ M. Imada, A. Fujimori, and Y. Tokura, Rev. Mod. Phys. 70, 1039 (1998).

${ }^{4}$ P. G. de Gennes, Phys. Rev. 118, 141 (1960), and references therein.

${ }^{5}$ A. J. Millis, Phys. Rev. B 53, 8434 (1996); Nature (London) 392, 147 (1998).

${ }^{6}$ E. Müller-Hartmann and E. Dagotto, Phys. Rev. B 54, R6819 (1996)

${ }^{7}$ P. Wagner, I. Gordon, L. Trappeniers, J. Vanacken, F. Herlach, V. V. Moshchalkov, and Y. Bruynseraede, Phys. Rev. Lett. 81, 3980 (1998).

${ }^{8}$ P. G. Radaelli, D. E. Cox, M. Marezio, and S.-W. Cheong, Phys. Rev. B 55, 3015 (1997).

${ }^{9}$ H. Kuwahara, Y. Tomioka, A. Asamitsu, Y. Moritomo, and Y. Tokura, Science 270, 961 (1995).

${ }^{10}$ For a review of Wigner crystallization, see C. M. Care and N. H. March, Adv. Phys. 24, 101 (1975).

${ }^{11}$ Y. Tomioka, A. Asamitsu, Y. Moritomo, H. Kuwahara, and Y. Tokura, Phys. Rev. Lett. 74, 5108 (1995).

${ }^{12}$ H. Kawano, R. Kajimoto, H. Yoshizawa, Y. Tomioka, H. Kuwahara, and Y. Tokura, Phys. Rev. Lett. 78, 4253 (1997).

${ }^{13}$ L. Pinsard, J. Rodriguez-Carvajal, A. H. Moudden, A. Anane, A. Revcolevschi, and C. Dupas, Physica B 234-236, 856 (1997).

${ }^{14}$ S. Uhlenbruck, R. Teipen, R. Klingeler, B. Büchner, O. Friedt, M. Hücker, H. Kierspel, T. Niemöller, L. Pinsard, A. Revcolevschi, and R. Gross, Phys. Rev. Lett. 82, 185 (1999).

${ }^{15}$ T. Niemöller, M. v. Zimmermann, J. R. Schneider, S. Uhlenbruck, B. Büchner, T. Frello, N. H. Andersen, L. Pinsard, A. M. de Léon-Guevara, and A. Revcolevschi, Eur. Phys. J. B 8, 5 (1999).

${ }^{16}$ G. A. Gehring and K. A. Gehring, Rep. Prog. Phys. 38, 1 (1995).

${ }^{17}$ J. B. Goodenough, A. Wold, R. J. Arnott, and N. Menyuk, Phys. Rev. 124, 373 (1961); J. B. Goodenough and J. M. Longo, in Crystallographic and Magnetic Properties of Perovskite and Perovskite-related Materials, edited by K.-H. Hellwege, Landolt-Börnstein, New Series Group III, Vol. 4, Pt. A (Springer-Verlag, Berlin, 1970), p. 126.

${ }^{18}$ Y. Murakami, J. P. Hill, D. Gibbs, M. Blume, I. Koyama, M.
Tanaka, H. Kawata, T. Arima, Y. Tokura, K. Hirota, and Y. Endoh, Phys. Rev. Lett. 81, 582 (1998).

${ }^{19}$ J. Rodriguez-Carvajal, M. Hennion, F. Moussa, L. Pinsard, and A. Revcolevschi, Physica B 234-236, 848 (1997).

${ }^{20}$ A. Anane, C. Dupas, K. Le Dang, J. P. Renard, P. Veillet, A. M. de Léon-Guevara, F. Millot, L. Pinsard, and A. Revcolevschi, J. Phys. Condens. Matter 7, 7015 (1995), and references therein.

${ }^{21}$ F. Herlach, C. C. Agosta, R. Bogaerts, W. Boon, I. Deckers, A. De Keyzer, N. Harrison, A. Lagutin, L. Li, L. Trappeniers, J. Vanacken, L. Van Bockstal, and A. Van Esch, Physica B 216, 161 (1996).

${ }^{22}$ H. Kawano, R. Kajimoto, M. Kobota, and H. Yoshizawa, Phys. Rev. B 53, R14 709 (1996).

${ }^{23}$ J. J. Neumeier, K. Andres, and K. J. McClellan, Phys. Rev. B 59, 1701 (1999).

${ }^{24}$ A. Anane, C. Dupas, K. Le Dang, J. P. Renard, P. Veillet, L. Pinsard, and A. Revcolevschi, Appl. Phys. Lett. 69, 1160 (1996).

${ }^{25}$ A. Moreo, S. Yunoki, and E. Dagotto, Science 283, 2034 (1999).

${ }^{26}$ A. L. Efros and B. I. Shklovskii, J. Phys. C 8, 249 (1975).

${ }^{27}$ F. G. Aliev, E. Kunnen, K. Temst, K. Mae, G. Verbanck, J. Barnas, V. V. Moshchalkov, and Y. Bruynseraede, Phys. Rev. Lett. 78, 134 (1997), and references therein.

${ }^{28}$ T. A. Tyson, J. Mustre de Leon, S. D. Conradson, A. R. Bishop, J. J. Neumeier, H. Röder, and Jun Zang, Phys. Rev. B 53, 13985 (1996).

${ }^{29}$ R. Maezono, S. Ishihara, and N. Nagaosa, Phys. Rev. B 57, R13 993 (1998).

${ }^{30} \mathrm{~A}$ different low-temperature orbital structure, based on resonant $\mathrm{X}$-ray scattering data and invoking a hybridization of $3 z^{2}-r^{2}$ and $x^{2}-y^{2}$ orbitals, was recently suggested by Y. Endoh et al., Phys. Rev. Lett. 82, 4328 (1999).

${ }^{31}$ J. Fontcuberta, B. Martínez, A. Seffar, S. Piñol, J. L. GarcíaMuñoz, and X. Obradors, Phys. Rev. Lett. 76, 1122 (1996).

${ }^{32}$ N. F. Mott and E. A Davis, Electronic Processes in NonCrystalline Materials (Clarendon Press, Oxford, 1979).

${ }^{33}$ J. M. D. Coey, M. Viret, L. Ranno, and K. Ounadjela, Phys. Rev. Lett. 75, 3910 (1995).

${ }^{34}$ J. M. De Teresa, M. R. Ibarra, P. A. Algarabel, C. Ritter, C. Marquina, J. Blasco, J. Garcia, A. Delmoral, and Z. Arnold, Nature (London) 386, 256 (1997).

${ }^{35}$ O. Chauvet, G. Goglio, P. Molinie, B. Corraze, and L. Brohan, Phys. Rev. Lett. 81, 1102 (1998).

${ }^{36}$ N. W. Ashcroft and N. D. Mermin, Solid State Physics (Saunders College, Philadelphia, 1976).

${ }^{37}$ B. I. Shklovskii and A. L. Efros, Electronic Properties of Doped Semiconductors, Springer Series in Solid-State Sciences Vol. 45 (Springer-Verlag, Berlin, 1984). 